Ann. Parasitol. Hum. Comp., 1990, 65, Suppl. I : 89-93.
Mots-clés: Maladie du sommeil. Trypanosoma gambiense. Contrôle. Dépistage.

Key-words: Sleeping sickness. Trypanosoma gambiense. Control. Screening.

\title{
LES LENTS PROGRÈS DU CONTRÔLE DE LA MALADIE DU SOMMEIL
}

\author{
M. WÉRY
}

\begin{abstract}
RÉSUMÉ
Depuis presqu'un siècle, les mêmes méthodes sont utilisées pour essayer de contrôler les foyers de trypanosomiase à $T$. gambiense. Les progrès techniques se résument à l'introduction du diagnostic sérologique avec un net gain en sensibilité par rapport à la palpation ganglionnaire, à l'amélioration des méthodes de concentration parasitaire en vue de la confirmation du diagnostic et à la simplification des méthodes efficaces de piégeage des glossines.

Cependant, aucune de ces méthodes, aussi simplifiées, soient-elles, n'est aisément applicable dans les conditions de terrain dans lesquelles se transmet $T$. gambiense. Des progrès importants en biologie moléculaire du trypanosome ont été faits ces dernières années, mais aucune retombée n'a été enregistrée jusqu'à présent dans le domaine de la détection active des cas ni dans le traitement des patients ou des porteurs sains.
\end{abstract}

\section{Summary: Sluggish improvements in the control of sleeping sickness.}

The century-old controls methods of $T$. gambiense infection foci have not been changed significantly in the past decennias. The technical improvements are restricted to (1) the introduction of serological diagnosis bringing a marked gain in sensitivity as compared to lymphnode palpation, (2) new parasite concentration methods for corroborating the diagnosis and (3) the design of cheap and efficient traps for glossina. None of these methods however, although smooth to perform and plain in design, is easily applied in the field conditions where T. gambiense is transmitted. Progress in the molecular biology of the trypanosome has been booked in the last few years, but it does not yet bring about a change in the active detection and the treatment of the patients and parasite carriers.
Le titre est restrictif : ce qui suit concerne l'infection à Trypanosoma brucei gambiense. La problématique de son contrôle aura été un souci en Afrique pendant tout le vingtième siècle.

\section{LA PREMIÈRE DÉCENNIE}

Les missions envoyées en Afrique Centrale au début du siècle avaient pris conscience de l'existence de la maladie du sommeil, de ses caprices épidémiques et des extensions endémiques liées aux mouvements de population. La gravité de la maladie exigeait la promulgation de mesures policières pour éviter les catastrophes épidémiques. Les parasites responsables avaient été identifiés en $T$. brucei, $T$. rhodesiense et $T$. gambiense et leurs principales caractéristiques reconnues (Laveran et Mesnil, 1912).

Des équipes de médecins, pourtant d'une grande perspicacité, avaient reconnu l'absence de symptômes facilement décelables au cours de la première phase extrêmement

Institut de Médecine tropicale, 155, Nationalestraat, B-2000 Antwerpen. longue, dite hémolymphatique, de l'infection par T. gambiense. Ils avaient donc implicitement reconnu l'existence de porteurs sains de trypanosomes et localisé le principal réservoir de parasites (Martin et al., 1908).

Ils avaient noté que les adénopathies, seul signe extérieur de cette phase débutante n'étaient présentes que chez la moitié des malades diagnostiqués par la mise en évidence du parasite.

Ils vivaient, déjà à l'époque, dans la hantise de voir les patients rechuter après un traitement pourtant bien conduit avec l'Atoxyl ou le Tartre émétique, trypanocides imparfaits et toxiques.

Les mesures préventives destinées à limiter l'extension des foyers endémiques comprenaient (Dutton et Todd, 1906) :

- le dépistage chez les voyageurs le long des routes par palpation des ganglions cervicaux, suivi de l'examen microscopique de la lymphe ganglionnaire et du sang;

- la mise en traitement immédiate des sujets infectés pour stériliser le sang périphérique;

- le regroupement des malades dans des hôpitaux à l'abri des glossines; 
- le déplacement des villages loin des gîtes à glossines ou le débroussaillement autour des lieux habités pour limiter le contact homme-glossine.

Le réservoir animal avait été évoqué sans qu'on ne puisse faire un début d'inventaire des espèces animales réceptives au trypanosome ni déterminer chez elles la prévalence de l'infection.

Des générations de responsables de la santé ont « planché » sur la problématique du diagnostic précoce. Suivant la logique des connaissances acquises dès le début, des équipes mobiles de recensement médical ont été organisées par Jamot, innovateur dans ce domaine. Cependant, de tout temps, ceux qui se sont attelés à cette tâche et Jamot, le premier, ont pris des risques car, peu orthodoxes dans leurs méthodes, ils bousculaient la hiérarchie médicale et administrative et, audacieux en thérapeutique, ils s'exposaient par les accidents à la vindicte des populations qu'ils s'étaient promis de protéger.

\section{L'ENDÉMIE AU SEUIL DE LA DERNIÈRE DÉCENNIE}

L'endémie sommeilleuse en 1990 persiste à un niveau menaçant dans la plupart des pays africains. De nouveaux foyers apparaissent, d'autres sont difficilement contrôlés pendant des années pour régresser soudain sans que rien n'ait changé apparemment dans la manière de les traiter.

Les 10 millions de kilomètres carrés infestés par des glossines transmettant pêle-mêle trypanosomiases humaines et animales en savane, dans les forêts galeries et en forêt, sont le théâtre de fluctuations de l'endémie.

L'interprétation des chiffres cités dans les rapports doit être prudente. Le nombre de cas diagnostiqués par les services de dépistage actif dépend, certes de l'incidence, mais surtout du rendement des équipes chargées du recensement (Josseran, 1987) : deux manquements majeurs peuvent être la couverture incomplète de la population vulnérable et un absentéisme aux recensements qui peut dépasser $80 \%$.

\section{MOYENS TECHNIQUES ACTUELS}

Les progrès techniques récents ont amélioré les méthodes de diagnostic qui doivent être utilisables sur le terrain, car la trypanosomiase n'est pas une endémie urbaine.

La percée la plus importante est, sans aucun doute, la connaissance de la composition antigénique et particulièrement de l'antigène variable de nature glycoprotéique de la surface du trypanosome qui caractérise une population parasitaire. Dans l'organisme du patient, les populations se succèdent, issues de quelques mutants ayant survécu à la lyse immune de la population précédente. Ce comportement explique les fluctuations de la parasitémie et les variations dans le temps du rendement d'un examen de sang.
Chaque population présente un antigène différent qui lui permet d'échapper aux anticorps sériques.

Les gènes responsables de la synthèse de ces glycoprotéines sont connus (Dero et al., 1987). L'activation successive de parties du génome détermine la séquence des variants observés. Cette séquence, appelée répertoire antigénique est caractéristique d'un isolat (stock) de trypanosomes et a permis la mise au point de tests sérologiques basés sur l'identification des variants précoces et dominants. L'immunofluorescence indirecte (IFI), l'hémagglutination (Cellognost $^{\circledR}$ ) et l'agglutination directe sur carte ou CATT (Magnus et al., 1978), qui sont positives chez plus de $90 \%$ des trypanosés, reposent sur l'antigène de surface.

La mise en évidence du parasite dans le sang a vu son rendement augmenté par la centrifugation en capillaire de Woo et par la filtration sur cellulose (mAECT de Lumsden). Des nécessaires prêts à l'emploi sont disponibles pour utilisation dans les laboratoires de terrain rudimentaires (Wéry et Mulumba, 1989).

La lomidinisation prophylactique a été abandonnée; le traitement par l'Arsobal reste le seul valable en toutes circonstances, malgré sa toxicité (Wéry et Burke, 1989).

\section{LE DÉPISTAGE ACTIF PAR ÉQUIPES MOBILES}

Les quatre étapes du dépistage restent le tri, la confirmation, la détermination du stade et le traitement.

Le tri se base forcément soit sur un signe clinique facile à rechercher soit sur une méthode de laboratoire rapide, sensible, spécifique, simple à réaliser et bon marché. La palpation des ganglions cervicaux nécessite les dix doigts compétents d'un infirmier expérimenté. Le CATT nécessite un minimum de matériel et du personnel précis. Il s'agit de distinguer les vrais agglutinats souvent discrets des faux précipités d'un antigène mal conservé. Dans la bousculade des recensements les mieux organisés, l'identification de la personne, le prélèvement, la numérotation des spots sur la carte, la notification du résultat, tout est délicat. La table bancale, le tabouret inconfortable et le risque, surtout pour les examens en grande série, de dessiccation par le soleil, dans la fournaise sous un toit de tôles, ou de dilution intempestive par une goutte de pluie, peuvent compromettre la lecture.

Les suspects identifiés par le tri seront gardés à vue pour la recherche du parasite au microscope, dans la lymphe ganglionnaire ou dans le sang examiné à frais ou, si ce luxe est possible, après concentration parasitaire. Les ponctions lombaires font partie de la routine et terminent traditionnellement la journée de recensement. Elles concernent les malades identifiés et les anciens cas sous surveillance post-thérapeutique. Il n'y aura plus alors qu'à pratiquer l'injection de Suramine ou de Lomidine stérilisante pour le sang périphérique et à convoquer les malades identifiés à l'hôpital ou au centre de traitement spécialisé. 
Il est cependant difficile de concilier la répétition des examens microscopiques du sang nécessaires à une bonne sensibilité avec le nomadisme de l'équipe condamnée à continuer sa route une fois les examens terminés, avant la tombée de la nuit. Restent les séropositifs qui n'auront pas été confirmés du premier coup : on en compte souvent plus de $50 \%$ et les microscopistes gardent l'impression d'un travail inachevé.

\section{ALTERNATIVES POSSIBLES UX ÉQUIPES MOBILES}

Trois éléments se conjuguent actuellement pour faire baisser la motivation des autorités sanitaires à consentir l'effort d'organiser un coûteux et exigeant recensement par équipes mobiles : l'augmentation de la couverture par les zones de santé, le perfectionnement des méthodes de la lutte antivectorielle et la publicité faite à propos d'un réservoir animal.

\section{Les zones de santé}

Les programmes «Santé pour tous» ont promu les soins de santé de première ligne, avec comme but final l'accessibilité à un centre de santé pour l'entièreté de la population. Il paraît donc normal de confier le dépistage de la trypanosomiase aux responsables des zones de santé.

Les infirmiers des centres de santé ne peuvent pas se déplacer et ne peuvent donc pas faire du dépistage actif. Ils doivent se contenter de s'entraîner à faire avouer des plaintes omniprésentes à force d'être banales comme des maux de tête, des sensations de chaleur à la tête, des douleurs, fourmillement ou chaleur aux plantes des pieds, aux paumes des mains ou au bout des doigts et des orteils, de la fatigue, une somnolence diurne et des démangeaisons. Ces plaintes doivent être perçues depuis plus de 30 jours.

Ils doivent s'exercer à rechercher systématiquement chez tout consultant les adénopathies cervicales, dont le caractère typique est souvent difficile à apprécier, surtout chez les enfants.

Ils peuvent généraliser, dans les zones endémiques, l'emploi d'un test sérologique, en y soumettant périodiquement l'entièreté de la population sous leur juridiction ou, de manière plus réaliste, un échantillon témoin de cette population (les systèmes vigies). Dans cet échantillon représentatif et composé de personnes plus ou moins volontaires, toute augmentation de l'incidence sérologique servira d'alerte déclenchant une action de dépistage spécialisé. La surcharge d'un travail qui se doit minutieux et le sacrifice budgétaire sont malheureusement peu compatibles avec un système autofinancé.

Les méthodes les plus simples comme le CATT présentent malgré tout des difficultés de réalisation et des aléas de lecture. La nécessité de réfrigération des réactifs, l’obli- gation de disposer d'un agitateur rotatif, les hésitations bien compréhensibles lors de l'interprétation par du personnel polyvalent, le prix d'un test sont autant d'obstacles à l'utilisation systématique de la sérologie dans les centres de santé. Pour l'immunofluorescence indirecte, les prélèvements sont envoyés à un laboratoire central : la connaissance du résultat est différée, les possibilités d'erreurs d'identification sont multipliées et la motivation du personnel est diminuée aux deux extrémités de la chaîne.

Une fois le suspect repéré, la confirmation du diagnostic pose les mêmes problèmes que pour l'équipe mobile : mise en œuvre des techniques de concentration et répétition des examens. Reste à convaincre le suspect bien portant de revenir et le microscopiste de faire des heures supplémentaires...

\section{La simplification des méthodes de lutte nti-vectorielle}

Le choix de cette approche de lutte implique la prolongation indéfinie de son application.

Les insecticides ont été abandonnés. Leur application relevait en forêt de la performance sportive et en savane, où les applications par avion sont possibles, de la prouesse financière.

Les mâles stériles qui ont donné lieu à des essais bien contrôlés ne se plient à aucune recette standard et restent un défi, car la production commerciale relève de la science fiction et le lâcher dans un biotope exige des études entomologiques longitudinales et augmente transitoirement la population vectrice.

Le piégeage pourrait être confié aux habitants eux-mêmes à condition qu'ils sachent à quoi il sert. Les principes en sont simples : les glossines sont éliminées en les emprisonnant dans un piège ou en interposant sur leur passage un écran de tissu imprégné d'insecticide. Dans les deux cas, il faut que l'insecte soit attiré par le dispositif. D'où les études nombreuses alliant l'originalité et le folklore, jouant sur la couleur, la forme et le mouvement. On a vu des pièges reproduisant des formes animales, on a entendu le frou-frou des jupes longues en tissu sombre ajustées à la taille de captureurs qui déambulaient aux endroits propices. Le piège biconique blanc-bleu (Challier, 1977) et le pyramidal bleu-noir (Gouteux et Lancien, 1986) ont été reconnus les plus efficaces et les plus faciles à fabriquer, avec leur armature simple habillée de tissu. Leur prix et la difficulté de leur entretien sont deux obstacles à leur généralisation.

Les écrans, simples morceaux de tissu, imprégnés de produits capables de tuer les mouches constituent peut-être l'ultime simplification. Des études récentes très fouillées font appel à la chimie des fibres textiles, des colorants et des insecticides pour réunir les quatre qualités recherchées : attractivité, résistance aux intempéries, bonne imprégnation et rémanence. 
Les mélanges polyester-coton ont la préférence, pour leur résistance à l'usure, la stabilité de leur coloration et leur capacité de retenir une quantité efficace d'insecticide.

Pour la couleur, on hésite encore : le bleu électrique attire le mieux les mouches, mais elles préfèrent se poser sur du noir (Laveissière, 1987). On hésite aussi sur la nécessité de combiner deux couleurs plus ou moins contrastées et sur la manière de les juxtaposer.

Les insecticides en concentrés émulsifiables sont les meilleurs. Plutôt que les organochlorés trop vite inactivés et trop lents à tuer les mouches, on préfère les pyréthrinoïdes, l'alphaméthrine (200 mg de concentré émulsifiable par mètre carré) étant jugée supérieure à la deltaméthrine (Laveissière, 1985). Les mouches qui viennent se poser sur les écrans doivent être intoxiquées en moins de 10 secondes.

Un écran imprégné résiste pendant 3 à 4 mois, mais en saison des pluies, la rémanence tombe à 45 jours au maximum. Les réimprégnations constituent une manipulation coûteuse et encombrante.

En conclusion, le piégeage qui a pu réduire de plus de $95 \%$ la population initiale de mouches dans certaines plantations entretenues, reste une méthode coûteuse vu son extension prohibitive dans le temps et l'espace. Quant à la motivation des villageois, elle est généralement de courte durée si elle a pu être suscitée à un moment. Les écrans ou les pièges sont alors abandonnés à l'agressivité du milieu écologique qui les détruit : soleil, pluie, poussière. C'est pourtant une méthode qui pourrait résoudre le problème des zones éternellement endémiques.

\section{Le réservoir de $\mathrm{T}$. b. gambiense}

La virulence très basse de $T$. $b$. gambiense en fait une espèce difficile à étudier en laboratoire : isolément laborieux à partir des hôtes naturels et adaptation problématique à des animaux de laboratoire, cobayes, rats, souris (Wéry et al., 1977). Il était donc admis qu'il ne pouvait subsister que chez l'homme, et que le malade en était l'unique réservoir. D'où l'énergie déployée pour faire un diagnostic aussi précoce que possible des sujets infectés. Il est cependant important de ne pas perdre de vue l'existence de porteurs sains qui peuvent être repérés par les méthodes sérologiques. Leur existence a été prouvée par de nombreux auteurs.

Chez des animaux, la présence de $T$. vivax, $T$. congolense et $T . b$. brucei, plus virulents et mieux adaptés à leur hôte peut masquer la présence éventuelle de T. b. gambiense. Mais le principal obstacle réside dans l'impossibilité de distinguer au microscope $T . b$. brucei de $T$. b. gambiense. Il a donc fallu attendre la mise au point de méthodes capables de différencier les 3 sous-espèces de $T$. b. brucei pour obtenir une information utile.

Mehlitz (1986), dans plusieurs pays d'Afrique de l'Ouest, isole à partir de porcs et de chiens plusieurs stocks de T. b. gambiense identifiés par les caractères de leurs isoenzymes selon la technique décrite par Godfrey et al. (1976). Noireau et al. (1989) ont identifié T. b. gambiense chez plusieurs espèces animales en République Populaire du Congo en utilisant l'hybridation de l'ADN des parasites associée à la taxonomie numérique (Paindavoine et al., 1986). Au Zaïre, Kageruka (1989) a isolé deux souches assimilables à $T$. b. gambiense chez la chèvre et le porc, parmi de beaucoup plus nombreux autres isolats de même morphologie identifiés comme étant $T$. b. brucei.

Les efforts considérables consentis ces dernières années et la publicité faite au moindre isolat de $T$. b. gambiense d'origine animale, comme pour se donner une excuse à la persistance des foyers, ne sont pas convaincants pour les épidémiologistes : les animaux vivant dans et autour des villages ne joueraient pas un rôle significatif comme réservoir de l'infection humaine. Cependant, ces bruits persistants de réservoir animal finissent par enlever une partie de la motivation des équipe mobiles et des autorités sanitaires pour continuer le dépistage.

\section{PERSPECTIVES NOUVELLES}

\section{La connaissance $d u$ parasite}

Des caractères biochimiques (les enzymes digestifs) et génétiques (hybridation d'ADN) déjà évoqués à propos du réservoir animal permettent depuis peu de distinguer les unes des autres les sous-espèces de $T$. brucei.

La difficulté de disposer de $T$. $b$. gambiense au laboratoire est en passe d'être levée, grâce à deux approches nouvelles : le xénodiagnostic et la culture. Il serait temps que la trajectoire de ce parasite insaisissable soit enfin vérifiée et son identité précisée, alors que des auteurs font état de l'infection humaine par un $T$. "non gambiense ", en Afrique de l'Ouest (Gibson, 1986).

Dukes et al. (1989) viennent d'enregistrer $75 \%$ de succès en nourrissant des glossines d'élevages avec du sang de patients expédié au laboratoire spécialisé sous forme congelée. Les formes procycliques de l'estomac de la glossine sont ensuite cultivées in vitro pour produire sans difficulté majeure de grandes quantités de parasites. Des infections de glandes salivaires peuvent également être obtenues et des populations de trypanosomes métacycliques, source de l'infection chez l'homme pourront être mieux étudiées.

La culture des formes sanguicoles est également singulièrement facilitée par la méthode « KIVI» (kit for in vitro isolation). Des flacons type pénicilline scellés et contenant un milieu GLSH (glucose, lactalbumine, sérum, hémoglobine) modifié et additionné d'antibiotique, sont envoyés sur le terrain. Du sang du patient est prélevé stérilement grâce à un veinotube hépariné et immédiatement injecté dans le flacon de culture à travers le bouchon. Une fois le prélèvement dans le flacon, tout est fait. L'acheminement au laboratoire ne nécessite aucune précaution. Sauf 
surinfection, les trypanosomes se multiplient à la température ambiante en 3 à 5 semaines, avec un rendement dépassant $75 \%$ des prélèvements (Le Ray, communication personnelle).

Les retombées pour le dépistage sont néanmoins encore lointaines; la difficulté du diagnostic parasitologique chez un malade reste actuellement le grand handicap, associée à la toxicité du traitement par l'Arsobal.

\section{Les thérapeutiques nouvelles}

En 1947, l'Arsobal (melarsenoxide) a pris le relais du Tryparsamide comme médicament pouvant accéder à tous les recoins de l'organisme où peut se cacher le trypanosome. Il est toujours le seul aujourd'hui à pouvoir rendre ce service éminent (Wéry et Burke, 1989). Malheureusement, sa réputation de toxicité n'est pas surfaite et la synthèse de cette molécule arsénicale pose des problèmes aux laboratoires qui en assurent la production, uniquement sur commande, d'où son prix prohibitif pour les pays endémiques.

Un médicament efficace et peu toxique réglerait le problème du réservoir de trypanosomes en autorisant le traitement immédiat des séropositifs. Trois familles chimiques présentent des candidats : les nitrofuranes avec le lévofuraltadone et le nifurtimox (Lampit), les dérivés azolés avec le benznidazole (Radanil), et les inhibiteurs de l'ornithine décarboxylase avec la difluoro-méthyl ornithine (Eflornitine). Tous ont à un moment, été essayés avec succès dans le traitement de cas résistants à l'Arsobal à différents stades d'évolution. Mais aucun n'est, jusqu'à présent, accessible pour un traitement régulier de cas fraîchement diagnostiqués. On a l'impression de tourner en rond et d'organiser toujours plus d'essais chez des cas désespérés avec des résultats forcément inconstants. Le DFMO (Eflornithine) est à l'essai depuis 8 ans et aucune décision n'a été prise à son sujet. Il est vrai que dans sa forme actuelle, il faut $1 \mathrm{~kg}$ de cette poudre blanche pour traiter un malade. A quand un progrès décisif dans ce domaine crucial?

\section{RÉFÉRENCES}

Challier A., Eyrand M., Lafaye A., Laveissière C. : Amélioration du rendement du piège biconique pour glossines (Diptera, Glossinidae) par l'emploi d'un cône inférieur bleu. Cah. ORSTOM [sér. Entomol. méd. et Parasitol.], 1977, 15, 283-386.

Dero B., Zampetti-Bosseler F., Pays E., Steinert M. : The genome and the antigen repertoire of Trypanosoma brucei gambiense are smaller than those of T. b. brucei. Mol. Biochem. Parasitol., 1987, 26, 247-256.
Dukes P., Kaukas A., Hudson K. M., Asongani T., Gashuma J. K. : A new method for isolating Trypanosoma brucei gambiense from sleeping sickness patients. Trans. Roy. Soc. Trop. Med. Hyg., 1989, 83, 636-639.

Dutton J. E., Todd J. L. : The distribution and spread of sleeping sickness in the Congo Free State with sugggestions on prophylaxis. Liverpool School of Tropical Medicine, 1906, Mem. XVIII.

Gibson W. C. : Will the real Trypanosoma b. gambiense please stand up. Parasitol. Today, 1986, 2, 255-257.

Godfrey D. G., Kilgour V. : Enzyme electrophoresis in characterizing the causative organism of gambian trypanosomiasis. Trans. Roy. Soc. Trop. Med. Hyg., 1976, 70, 219-224.

Gouteux J. P., Lancien J. : Le piège pyramidal à tsé-tsé (Diptera : Glossinidae) pour la capture et la lutte. Essais comparatifs et description de nouveaux systèmes de capture. Trop. Med. Parasitol., 1986, 37, 61-66.

Josseran R., Hengy C., Calvez Th., Kouka Bemba D. : Évolution de la trypanosomiase humaine dans les pays de l'OCEAC. Med. Trop. Coop. Allo Sviluppo, 1987, 3, 62-66.

Laveissière C., Couret D., Manno A., Küpper W. : Tests d'efficacité d'insecticides utilisés en imprégnation sur tissus pour la lutte par piégeage contre les glossines. 2. Première série de tests en saison sèche. Cah. ORSTOM [sér. Entomol. méd. et Parasitol.], 1985, 23, 217-230.

Laveissière C., Couret D., Grébaut P. : Recherche sur les écrans pour la lutte contre les glossines en région forestière de Côted'Ivoire. Mise au point d'un nouvel écran. Cah. ORSTOM [sér. Entomol. méd. et Parasitol.], 1987, 25, 145-164.

Laveran A., Mesnil F. : Trypanosomes et trypanosomiases. Masson et Cie, éditeurs, Paris, 1912.

Magnus E., Vervoort E., Van Meirvenne N. : A card-agglutination test with stained trypanosomes (C. A. T. T.) for the serological diagnosis of T. b. gambiense. Ann. Soc. Belge Med. Trop., 1978, 58, $169-176$.

Martin G., Lebœuf A. : Période d'incubation dans la maladie du sommeil. Inflammations locales à la suite de piqûres de glossines infectées. Bull. Soc. Pathol. Exot., 1908, 1, 7.

Mehlitz D. : Le réservoir animal de la maladie du sommeil à Trypanosoma brucei gambiense. Études et synthèses de l'IEMVT (GTZ Germany, Maisons-Alfort, France), 1986, 18, 1-156.

Noireau F., Paindavoine P., Lemesre J. L., Toudic A., Pays E., Gouteux J. P., Steinert M., Frézil J. L. : The epidemiological importance of the animal reservoir of Trypanosoma gambiense in the Congo. 2. Characterization of the Trypanosoma brucei complex. Tropenmed. Parasit., 1989, 40, 9-11.

Paindavoine P., Pays E., Laurent M., Geltmeyer Y., Le Ray D., Mehlitz D., Steinert M. : The use of DNA hybridization and numerical taxonomy in determining relationship between Trypanosoma brucei stocks and subspecies. Parasitology, 1986, 92, 31-50.

Wéry M., Burke J. : Chemotherapy of human african trypanosomiasis. J. Chemother., 1989, suppl. ${ }^{\circ} 4$, 962-964.

Wéry M., Mulumba M. P. : Detection of Trypanosoma brucei gambiense in the host and alternative sleeping sickness diagnostic approaches. Ann. Soc. Belge Méd. Trop., 1989, 69, suppl. $\mathrm{n}^{\circ} 1,181-187$.

Wéry M., Weyn J., Ngimbi N. P., Colaert J. : Isolement de souches de $T$. gambiense au Zaire et leur adaptation aux animaux de laboratoire. Ann. Soc. Belge Méd. Trop., 1977, 57, 425-437. 